\title{
Too much chemotherapy
}

\author{
Fiona Godlee editor in chief
}

The BMJ

People with cancer are living longer now than 40 years ago. This is clearly good news. But how much of this improvement can we attribute to drug treatment? Not much, concludes Peter Wise this week in an article I humbly suggest all oncologists should read (doi:10.1136/bmj.i5792). The nearly $20 \%$ improvement in five year survival over the past four decades is probably mainly due to improved early diagnosis and treatment rather than developments in cytotoxic chemotherapy, he says. And patients are being badly misled by over-enthusiastic accounts of what chemotherapy can achieve. Many expect a cure. In reality they will gain on average only a few months of extra life.

The roots of this over-enthusiasm are sadly familiar but worth recounting in the specific highly charged and intensive context of cancer. Wise, a medical ethics consultant and former consultant physician, doesn't mince his words. Unrepresentative, industry funded trials that use surrogate endpoints are part of the problem. So too are regulatory failures, perhaps explained by regulatory capture in which "the regulator risks being regulated by the industry that it has been appointed to regulate."

Unjustified enthusiasm for cancer drug treatments comes at huge cost, financial and personal (including treatment related deaths and reduced quality of life), and increased risk of dying in hospital rather than at home. Many patients don't realise that opting for supportive rather than active treatment—often called "refusal"-is an option and may give them longer as well as better quality life than chemotherapy. Conflicts of interest among clinicians compound their reluctance to have tricky conversations.

If improved survival is indeed largely due to early diagnosis and treatment, how is this best achieved? Many patients visit their GP with vague or non-specific symptoms. How should these be investigated and followed up without causing unnecessary alarm or wasting precious resources? As Brian Nicholson and colleagues explain (doi:10.1136/bmj.i5515), "safety-netting" aims to ensure that patients don't drop through the healthcare net and are monitored until symptoms can be explained. But there is little evidence on whether this works or how to do it well. In particular, how much responsibility should patients be expected to take in chasing up and understanding test results? The authors encourage a sharing of this responsibility between clinicians and patients, but also clear communication and robust systems to prevent patients falling through the net.

Wise concludes with a call for higher bars for drug approval for new and existing drugs. Ethical cancer care demands empowerment of patients, he says, with accurate, impartial information followed by genuinely informed consent. And funds and attention should shift to prevention, early detection, prompt and radical treatment of localised and regional disease, and early provision of supportive care. Only then will cancer care serve patients rather than governments and industry. 